\title{
DEGENERACY STRUCTURE OF THE CALOGERO-SUTHERLAND MODEL: AN ALGEBRAIC APPROACH
}

\author{
N. Gurappa, Prasanta K. Panigrahi ${ }^{\dagger}$, V. Srinivasan* \\ School of Physics, University of Hyderabad, Hyderabad - 500 046, India
}

\begin{abstract}
The degeneracy structure of the eigenspace of the N-particle CalogeroSutherland model is studied from an algebraic point of view. Suitable operators satisfying $\mathrm{SU}(2)$ algebras and acting on the degenerate eigenspace are explicitly constructed for the two particle case and then appropriately generalized to the $N$-particle model. The raising and lowering operators of these algebras connect the states, in a subset of the degenerate eigenspace, with each other.
\end{abstract}

† E-mail: panisp@uohyd.ernet.in

* E-mail: vssp@uohyd.ernet.in 
The Calogero-Sutherland model (CSM) ${ }^{1}$ describing $N$, interacting, identical particles in one-dimension, have currently attracted wide interest, both in physics as well as mathematics literature. These exactly solvable quantum mechanical models have relevance to two-dimensional gravity, ${ }^{2}$ quantum Hall effect ${ }^{3}$ fractional statistics ${ }^{4}$ and a host of other problems of physical interest. ${ }^{5}$ For the model describing particles on a circle interacting via the inverse sine square potential, the basis set of the orthonormal eigenfunctions are the well-known Jack polynomials. ${ }^{6}$ These functions have been constructed recently, through an operator method, ${ }^{7}$ by making use of the $S_{N^{-}}$extended Heisenberg algebra. ${ }^{8}$ However, for particles on a line, interacting through pair-wise inverse square potential in the presence of harmonic confinement, the complete set of orthonormal states is yet to be constructed explicitly. In light of the degeneracy of this model, it is of considerable interest to provide operators that commute with the Hamiltonian and connect the members of the degenerate eigenspace (DE). One would also like to unravel the underlying algebraic structure of these operators.

For the two particle case, this problem has been recently analyzed and the symmetry, associated with the degeneracy, has been shown to originate from a polynomial extension of the $\mathrm{SU}(2)$ algebra. ${ }^{9}$ In this paper, we first re-analyze the two particle CSM and show that the degeneracy can be described by a $\mathrm{SU}(2)$ algebra. The above method is then extended to the $N$-particle case, where we point out the existence of several copies of $S U(2)$ algebras. The raising and lowering operators of these algebras connect various sub-sectors of the DE. The advantage of this approach lies in the fact that, for more than two particles, the polynomial $S U(2)$ algebra becomes fairly complicated and unlike the $S U(2)$ case, one needs to find the unitary representations of this algebra individually for each case. However, we have been unable to find the complete set of operators, that will connect all the degenerate states with each other.

The CSM Hamiltonian is given by $(\hbar=\omega=m=1)$

$$
H=-\frac{1}{2} \sum_{i=1}^{N} \partial_{i}^{2}+\frac{1}{2} \sum_{i=1}^{N} x_{i}^{2}+\frac{g^{2}}{2} \sum_{\substack{i, j \\ i \neq j}}^{N} \frac{1}{\left(x_{i}-x_{j}\right)^{2}} .
$$


We work in a sector of the configuration space corresponding to a definite ordering of the particle coordinates: $x_{1} \leq x_{2} \leq \cdots \leq x_{N}$. After performing a similarity transformation (ST), $H$ takes the following form,

$$
\begin{aligned}
H^{\prime} & =Z^{-1} H Z \\
& =-\frac{1}{2} \sum_{i} \partial_{i}^{2}+\frac{1}{2} \sum_{i} x_{i}^{2}-\lambda \sum_{\substack{i, j \\
i \neq j}} \frac{1}{\left(x_{i}-x_{j}\right)} \partial_{i}
\end{aligned}
$$

where $Z=\prod_{i<j}^{N}\left[\left|\left(x_{i}-x_{j}\right)\right|^{\lambda}\left(x_{i}-x_{j}\right)^{\delta}\right]$, and $g^{2}=(\lambda+\delta)(\lambda+\delta-1)$. Here $\delta=0$ or 1 represents the choice of the quantization of the $N$-particle system as bosons or fermions respectively; we choose $\delta=0$ for convenience.

On symmetrized eigenstates, the above Hamiltonian $H^{\prime}$ is equivalent to another one, $\bar{H}$, which can be factorized by the raising and lowering operators of the $S_{N^{-}}$extended Heisenberg $\operatorname{algebra}(\mathrm{SEH}){ }^{8} \mathrm{SEH}$ is given by $\left[a_{i}, a_{j}\right]=\left[a_{i}^{\dagger}, a_{j}^{\dagger}\right]=0$ and $\left[a_{i}, a_{j}^{\dagger}\right]=\delta_{i j}\left\{1+\lambda \sum_{l} K_{i l}\right\}-\lambda K_{i j}$; where, the transposition operator $K_{i j}$ satisfies

$$
\begin{aligned}
K_{i j} & =K_{j i} ; \quad\left(K_{i j}\right)^{2}=1, \\
K_{i j} a_{j} & =a_{i} K_{i j} ; \quad K_{i j} a_{j}^{\dagger}=a_{i}^{\dagger} K_{i j} \quad \text { (no summation over repeated indices), } \\
K_{i j} K_{j l} & =K_{j l} K_{i l}=K_{i l} K_{i j}, \quad \text { for } i \neq j, i \neq l, j \neq l, \\
K_{i j} K_{m n} & =K_{m n} K_{i j}, \text { for } i, j, m, n \text { all different. }
\end{aligned}
$$

Explicitly,

$$
a_{i}\left(a_{i}^{\dagger}\right)=\frac{1}{\sqrt{2}}\left(x_{i}+(-) D_{i}\right)
$$

where,

$$
D_{i}=\partial_{i}+\lambda \sum_{\substack{j \\ j \neq i}}^{N} \frac{1}{\left(x_{i}-x_{j}\right)}\left(1-K_{i j}\right)
$$

is known as the Dunkl derivative. ${ }^{10}$ Now

$$
\begin{aligned}
\bar{H}=\frac{1}{2} \sum_{i}^{N}\left\{a_{i}, a_{i}^{\dagger}\right\} & =\left(-\frac{1}{2} \sum_{i}^{N} \partial_{i}^{2}+\frac{1}{2} \sum_{i}^{N} x_{i}^{2}-\lambda \sum_{\substack{i, j \\
i \neq j}}^{N} \frac{1}{\left(x_{i}-x_{j}\right)} \partial_{i}\right) \\
& +\frac{\lambda}{2} \sum_{\substack{i, j \\
i \neq j}}^{N} \frac{1}{\left(x_{i}-x_{j}\right)^{2}}\left(1-K_{i j}\right)
\end{aligned}
$$




$$
-\frac{\lambda^{2}}{2} \sum_{\substack{i, j, l \\ i \neq j, l}}^{N} \frac{1}{\left(x_{i}-x_{j}\right)}\left(1-K_{i j}\right) \frac{1}{\left(x_{i}-x_{l}\right)}\left(1-K_{i l}\right)
$$

and $\left[\bar{H}, a_{i}\left(a^{\dagger}\right)\right]=-a_{i}\left(a_{i}^{\dagger}\right)$. It is quite obvious that on symmetric states, the last two terms in Eq. (4) vanish identically and hence $\bar{H}=H^{\prime}$.

Recently, it has been shown in Ref. 9 that the degeneracy of the two particle CSM can be explained by a polynomial $S U(2)$ algebra. This was achieved by separating the relative and center of mass, creation and annihilation operators $A_{1}, A_{1}^{\dagger}$ and $A_{2}, A_{2}^{\dagger}$ respectively. The Hamiltonian in Eq. (1) can be written as

$$
H=\frac{1}{2}\left(\left\{A_{1}, A_{1}^{\dagger}\right\}+\left\{A_{2}, A_{2}^{\dagger}\right\}\right)=H_{1}+H_{2}
$$

where $\left[A_{1}, A_{1}^{\dagger}\right]=1+2 \lambda \sigma,\left[A_{2}, A_{2}^{\dagger}\right]=1$ and all other commutators vanish. Here $\sigma \equiv K_{12}$ anticommutes with $A_{1}, A_{1}^{\dagger}$ and commutes with $A_{2}$ and $A_{2}^{\dagger}$. Notice that, $\frac{1}{2} A_{1}^{2}, \frac{1}{2} A_{1}^{\dagger 2}$ and $\frac{1}{2} H_{1}$ generate a $S U(1,1)$ algebra; this is a part of the spectrum generating algebra of the two particle CSM. An un-normalized, generic, symmetric eigenstates can be written as

$$
\left|n_{1}, n_{2}>=A_{1}^{\dagger 2 n_{1}} A_{2}^{\dagger n_{2}}\right| 0>\quad,
$$

where $\mid 0>$ is the ground state satisfying $A_{1}\left|0>=A_{2}\right| 0>=0$. The generators of the polynomial $S U(2)$ algebra, which maps symmetric states into symmetric states are,

$$
\begin{aligned}
J_{o} & =\frac{1}{4}\left(A_{1}^{\dagger} A_{1}-A_{2}^{\dagger} A_{2}\right) \\
J_{+} & =\frac{\sqrt{\alpha}}{8}\left(A_{1}^{\dagger}\right)^{2} A_{2}^{2} \\
J_{-} & =\frac{\sqrt{\alpha}}{8}\left(A_{2}^{\dagger}\right)^{2}\left(A_{1}\right)^{2} .
\end{aligned}
$$

They commute with the Hamiltonian and satisfy

$$
\begin{aligned}
& {\left[J_{o}, J_{ \pm}\right]= \pm J_{ \pm}} \\
& {\left[J_{+}, J_{-}\right]=2 J_{o}-\alpha J_{o}^{3}-\beta J_{o}^{2}+\gamma}
\end{aligned}
$$

Here, $\alpha=32\left[N^{2}+2(\lambda+1) N+2\left(\lambda^{2}-1\right)\right]^{-1}, N=A_{1}^{\dagger} A_{1}+A_{2}^{\dagger} A_{2}, \gamma=\frac{1}{64} \lambda \alpha N(N+4)$ and $\beta=\frac{3}{4} \lambda \alpha$. 
For the above case, we first point out the method of construction of a regular $S U(2)$ algebra underlying the degeneracy. This is done by making use of the identities valid on the Fock space. ${ }^{11}$ This procedure is then generalized to the $N$ particle case.

In the following, we give the steps necessary for the construction of the $S U(2)$ generators. For the operators $F_{l}=A_{l}^{2}, l=1,2$, one can construct the canonical conjugates (CC) $G_{l}^{\dagger}$ : $\left[F_{l}, G_{l}^{\dagger}\right]=1$ (no summation over $l$ ) in several sectors. Explicitly, $G_{l}^{\dagger}$ can be written, in the vacuum sector as ${ }^{11}$

$$
G_{l}^{\dagger}=\frac{1}{2} F_{l}^{\dagger} \frac{1}{F_{l} F_{l}^{\dagger}}\left(A_{l}^{\dagger} A_{l}+2\right)
$$

The $S U(2)$ generators can then be defined as

$$
\begin{gathered}
J^{+}=G_{1}^{\dagger} F_{2}, J^{-}=G_{2}^{\dagger} F_{1} \\
J^{o}=\frac{1}{2}\left(G_{1}^{\dagger} F_{1}-G_{2}^{\dagger} F_{2}\right)=\frac{1}{2}\left(A_{1}^{\dagger} A_{1}-A_{2}^{\dagger} A_{2}\right)
\end{gathered}
$$

such that

$$
\begin{aligned}
& {\left[J^{0}, J^{ \pm}\right]= \pm J^{ \pm}} \\
& {\left[J^{+}, J^{-}\right]=2 J^{o}}
\end{aligned}
$$

It is straightforward to check that $J^{+}, J^{-}$and $J^{0}$ commute with $H$ and act in the degenerate space. The above procedure can be immediately generalized to the $N$-particle case. By choosing the center-of-mass, relative coordinates and their differential operators, respectively as,

$$
X=\frac{1}{N} \sum_{i} x_{i} ; y_{i}=x_{i}-X
$$

and

$$
\partial_{X}=\frac{1}{N} \sum_{i} \partial_{x_{i}} ; \partial_{y_{i}}=\partial_{x_{i}}-\partial_{X}
$$

$H^{\prime}$ in Eq. (2) can be written in the form

$$
\begin{aligned}
H^{\prime} & =\left(-\frac{N}{2} \partial_{X}^{2}+\frac{N}{2} X^{2}\right)+\left(-\frac{1}{2} \sum_{i}^{N} \partial_{y_{i}}^{2}+\frac{1}{2} \sum_{i} y_{i}^{2}-\lambda \sum_{\substack{i, j \\
i \neq j}}^{N} \frac{1}{\left(y_{i}-y_{j}\right)} \partial_{y_{i}}\right) \\
& =H_{X}+H_{\left\{y_{i}\right\}} .
\end{aligned}
$$


We define $A=\sqrt{\frac{N}{2}}\left(X+\partial_{X}\right)$ and $A^{\dagger}=\sqrt{\frac{N}{2}}\left(X-\partial_{X}\right)$ such that

$$
\left[A, A^{\dagger}\right]=1, H_{X}=\frac{1}{2}\left\{A, A^{\dagger}\right\} \quad,
$$

and

$$
\left[H_{X}, A\left(A^{\dagger}\right)\right]=-A\left(A^{\dagger}\right)
$$

$H_{\left\{y_{i}\right\}}$ can be factorized by making use of the SEH by replacing $x_{i}$ 's by $y_{i}{ }^{\prime}$ s in Eq. (4). The modified commutator is

$$
\left[a_{i}, a_{j}^{\dagger}\right]=\delta_{i j}\left\{1+\lambda \sum_{l} K_{i l}\right\}-\lambda K_{i j}-\frac{1}{N}
$$

and

$$
\bar{H}_{\left\{y_{i}\right\}}=\frac{1}{2} \sum_{i}\left\{a_{i}, a_{i}^{\dagger}\right\}
$$

It is easy to check that, $\left[A, a_{i}\right]=\left[A^{\dagger}, a_{i}\right]=\left[A^{\dagger}, a_{i}^{\dagger}\right]=\left[A^{\dagger}, a_{i}\right]=0$ and any completely symmetric state i.e., $K_{i j} \psi\left(\left\{y_{i}\right\}\right)=\psi\left(\left\{y_{i}\right\}\right)$ is a solution of both $\bar{H}_{\left\{y_{i}\right\}}$ and $H_{\left\{y_{i}\right\}}$. the ground-state $\mid 0>$ is annihilated by $a_{i}$ 's $i . e ., a_{i} \mid 0>=0$.

Keeping in mind the fact that, the spetrum and degeneracies of the CSM match identically with that of harmonic oscillators, one can choose $(N-1)$ completely symmetrized lowering and raising operators

$$
B_{n}=\sum_{i} a_{i}^{n} \quad ; B_{n}^{\dagger}=\sum_{i} a_{i}^{\dagger n}(N \geq n \geq 2)
$$

such that

$$
\left[\bar{H}_{\left\{y_{i}\right\}}, B_{n}\left(B_{n}^{\dagger}\right)\right]=-n B_{n}\left(n B_{n}^{\dagger}\right)(n \geq 2)
$$

For $n=1$ and 2, these operators are nothing but the elements of the harmonic oscillators and the $S U(1,1)$ algebras respectively. ${ }^{12}$ The $S U(2)$ algebra constructed above for the two body example can now be directly generalized to the $N$-particle case. We construct several non-commuting sets of $S U(2)$ algebras by making use of the fact that, $A$ and $A^{\dagger}$ commute 
with all the other $B_{n}$ and $B_{n}^{\dagger}$ operators, for arbitrary $n$. The CCs of $F_{n} \equiv A^{n}$ and $B_{n}$, obeying the following commutation relations

$$
\left[F_{n}, D_{n}^{\dagger}\right]=\left[B_{n}, G_{n}^{\dagger}\right]=1,
$$

can be respectively written in the vacuum sector as

$$
\begin{aligned}
D_{n}^{\dagger} & =\frac{1}{n} F_{n}^{\dagger} \frac{1}{F_{n} F_{n}^{\dagger}}\left(A^{\dagger} A+n\right), \\
G_{n}^{\dagger} & =\frac{1}{n} B_{n}^{\dagger} \frac{1}{B_{n} B_{n}^{\dagger}}\left(\sum_{i} a_{i}^{\dagger} a_{i}+n\right) .
\end{aligned}
$$

Using the above operators, the $S U(2)$ generators can be given as

$$
\begin{aligned}
J_{n}^{+} & =G_{n}^{\dagger} F_{n}, \\
J_{n}^{-} & =D_{n}^{\dagger} B_{n}, \\
\text { and } \quad J_{n}^{0} & =\frac{1}{2}\left(G_{n}^{\dagger} B_{n}-D_{n}^{\dagger} F_{n}\right)
\end{aligned}
$$

such that

$$
\begin{aligned}
& {\left[J_{n}^{0}, J_{n}^{ \pm}\right]= \pm J_{n}^{ \pm},} \\
& {\left[J_{n}^{+}, J_{n}^{-}\right]=2 J_{n}^{0}}
\end{aligned}
$$

Notice that, although the above generators commute with the Hamiltonian, they are mutually non-commuting due to the fact that $\left[B_{m}, B_{n}^{\dagger}\right] \neq 0$ for $m \neq n$. Hence, the raising and lowering operators of a given $S U(2)$ only connects the eigenstates of CSM in a given sub-sector of DE.

Since, we encountered the $B_{n}$ and $B_{n}^{\dagger}$ operators and the fact that $\left[B_{m}, B_{n}^{\dagger}\right] \neq 0$, it encourages us to study the algebraic structure underlying these operators. It is obvious that

$$
\left[\bar{H},\left[B_{n}, B_{n}^{\dagger}\right]\right]=\left[\bar{H}, W_{n, n}\right]=0
$$

and one can check that the operators $L_{n} \equiv W_{2, n+1}$ and $L_{m} \equiv W_{m+1,2}$ generate a centerless Virasoro algebra. Noticing that $\left[a_{i}, a_{j}^{\dagger}\right]=\left(\delta_{i j}-\frac{1}{N}\right)+\lambda \delta_{i j} \sum_{l} K_{i l}-\lambda K_{i j}$ in relative coordinates $y_{i}$, we first work out the $W_{m, n}$ commutation relations in $x_{i}$ coordinates, the coresponding 
relations with respect to $y_{i}$ 's can be established by replacing $\lambda$ independent $\delta_{i j}$ by $\delta_{i j}-\frac{1}{N}$. One easily checks that,

$$
\begin{aligned}
{\left[B_{m}, B_{n}^{\dagger}\right] } & =m \sum_{i}^{N} \sum_{r=0}^{n-1} a_{i}^{\dagger r} a_{i}^{m-1} a_{i}^{\dagger n-1-r} \\
& =n \sum_{i}^{N} \sum_{s=0}^{m-1} a_{i}^{s} a_{i}^{\dagger n-1} a_{i}^{m-1-s} \\
& \equiv W_{m n} \\
\text { and } \quad\left[\bar{H}, W_{m n}\right] & =(n-m) W_{m n}
\end{aligned}
$$

These $W_{m n}$ operators give a new non-linear basis for the $W_{\infty}$ algebra. First of all,

$$
\left[W_{m n}, a_{i}\left(a_{i}^{\dagger}\right)\right]=-m W_{m, n-1}^{(i)}\left(n W_{m-1, n}^{(i)}\right)+M_{i}
$$

where $M_{i}=\sum_{j}^{N} \sum_{k=0}^{m-1} \sum_{r=0}^{n-2} a_{j}^{k} a_{j}^{\dagger r} C_{i j} a_{j}^{\dagger n-2-r} a_{j}^{m-1-k}$ and $C_{i j}=\lambda\left\{\delta_{i j} \sum_{l}^{N} K_{i l}-K_{i j}\right\}$. Using the above equation, $W_{m n}$ 's commutation relations follows as given below:

$$
\left[W_{s-n-1, s+n-1}, W_{r-m-1, r+m-1}\right]=2\{n(r-1)-m(s-1)\} W_{s+r-2, n+m}+\cdots
$$

where $\cdots$ represent the coupling $\lambda, \hbar$ and $M_{i}$ dependent non-linear terms. This is a new realization of the $W_{\infty}$ algebra which differs from the earlier known ones. ${ }^{13}$

In conclusion, we reanalyzed the two particle CSM and constructed the $S U(2)$ algebra responsible for the degeneracy. This was generalized to the $N$-particle case and the existence of $N-1$ sets of non-commuting $S U(2)$ algebras was pointed out. In light of this, it is natural to suspect the existence of a higher symmetry algebra which contains all these $S U(2)$ 's as sub-algebras. In this respect, we arrived at a new $W_{\infty}$ algebra which differed from the earlier known ones. ${ }^{13}$ Since, this is a non-linear algebra, it is difficult to work out its representation theory. This strongly motivates us to search for the existence of an original dynamical algebra for CSM. One way out is to look for a linear $W_{\infty}$ algebra; work is in progress in this direction and will be published elsewhere.

\section{Acknowledgements}


N.G would like to thank Dr. J.S. Prakash and E. Harikumar for useful discussions and U.G.C (India) for the financial support through S.R.F scheme. 


\section{REFERENCES}

[1] F. Calogero, Jour. Math. Phys. 10, 2191 (1969); 12, 419 (1971); B. Sutherland, ibid. 12, $246(1971)$.

[2] I. Andric, A. Jevicki and H. Levine, Nucl. Phys. B 215, 307 (1983); A. Jevicki, ibid. B 376, 75 (1992).

[3] N. Kawakami, Phys. Rev. Lett. 71, 275 (1993); H. Azuma and S. Iso, Phys. Lett. B 331, 107 (1994); P.K. Panigrahi and M. Sivakumar, Phys. Rev. B 52, 13742 (15) (1995).

[4] F.D.M. Haldane, Phys. Rev. Lett. 67, 937 (1991); M.V.N. Murthy and R. Shankar, ibid, 73, 3331 (1994).

[5] For various connections, see the chart in B.D. Simons, P.A. Lee and B.L. Altshuler, Phys. Rev. Lett. 72, 64 (1994).

[6] R.P. Stanley, Adv. Math. 77, 76 (1988); I.G. Macdonald, Symmetric functions and Hall Polynomials (Clarendon Press, Oxford, 1995).

[7] L. Lapointe and L. Vinet, Commun. Math. Phys. 178, 425 (1996).

[8] M. Vasiliev, Int. J. Mod. Phys. A 6, 1115 (1991); A.P. Polychronakos, Phys. Rev. Lett. 69, 703 (1992); L. Brink, T.H. Hansson and M. Vasiliev, Phys. Lett. B 286, 109 (1992); L. Brink, T.H. Hansson, S.E. Konstein and M. Vasiliev, Nucl. Phys. B 384, 591 (1993); N. Gurappa and P.K. Panigrahi, Mod. Phys. Lett. A, 11, 891 (1996).

[9] R. Floreanini, L. Lapointe and L. Vinet, Phys. Lett. B 389, 327 (1996).

[10] C.F. Dunkl, Amer. Math. Soc. 311, 167 (1989).

[11] P. Shanta, S. Chaturvedi, V. Srinivasan and G.S. Agarwal, Phys. Rev. Lett. 72, 1447 (1994).

[12] A.M. Perelomov, Theor. Math. Phys. A6, 263 (1971); A.M. Olshanetsky and A.M. Perelomov, Phys. Rep. 71, 314 (1981); 94, 6 (1983). 
[13] K.H. Hikami and M. Wadati, Jour. Phys. Soc. Jpn. 62, 4203 (1993); ibid, Phys. Rev. Lett. 73, 1191 (1994); H. Ujino and M. Wadati, Jour. Phys. Soc. Jpn. 63, 3385 (1994; ibid, 64, 39 (1995); V. Narayanan and M. Sivakumar, Mod. Phys. Lett. A 11, 763 (1996); S.B. Isakov and J.M. Leinaas, preprint hep-th/9510184. 